\title{
Correction to: Testing assembly strategies of Francisella tularensis genomes to infer an evolutionary conservation analysis of genomic structures
}

Kerstin Neubert ${ }^{1,2}$, Eric Zuchantke ${ }^{3}$, Robert Maximilian Leidenfrost ${ }^{4}$, Röbbe Wünschiers ${ }^{4}$, Josephine Grützke ${ }^{2}$, Burkhard Malorny ${ }^{2}$, Holger Brendebach², Sascha Al Dahouk ${ }^{2}$, Timo Homeier ${ }^{5}$, Helmut Hotzel ${ }^{3}$, Knut Reinert ${ }^{1}$, Herbert Tomaso ${ }^{3}$ and Anne Busch ${ }^{3,6^{*}}$

Correction to: BMC Genomics 22, 822 (2021)

https://doi.org/10.1186/s12864-021-08115-x

Following publication of the original article [1], it was reported that Röbbe Wünschiers' name was misspelled as Roebbe Wuenschiers.

The original article [1] has been corrected.

\begin{abstract}
Author details
'Department of Mathematics and Computer Science, Algorithmic Bioinformatics, Freie Universität Berlin, Institute of Computer Science, Takustr. 9, 14195 Berlin, Germany. ${ }^{2}$ German Federal Institute for Risk Assessment, Diedersdorfer Weg 1, 12277 Berlin, Germany. ${ }^{3}$ Friedrich-Loeffler-Institut, Institute of Bacterial Infections and Zoonoses, Naumburger Str. 96a, 07749 Jena, Germany. ${ }^{4}$ Department of Biotechnology and Chemistry, Mittweida University of Applied Sciences, Technikumplatz 17a, 09648 Mittweida, Germany. ${ }^{5}$ Friedrich-Loeffler-Institut, Institute of Epidemiology, Südufer, 1017493 Greifswald, Insel Riems, Germany. ${ }^{6}$ Department of Anaesthesiology and Intensive Care Medicine, University Hospital Jena, Jena, Germany.
\end{abstract}

Published online: 14 December 2021

\section{Reference}

1. Neubert K, Zuchantke E, Leidenfrost RM, et al. Testing assembly strategies of Francisella tularensis genomes to infer an evolutionary conservation analysis of genomic structures. BMC Genomics. 2021;22:822. https://doi. org/10.1186/s12864-021-08115-x.

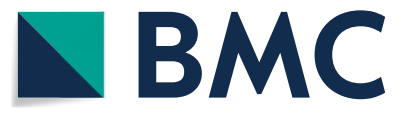
permits use, sharing, adaptation, distribution and reproduction in any medium or format, as long as you give appropriate credit to the original author(s) and the source, provide a link to the Creative Commons licence, and indicate if changes were made. The images or other third party material in this article are included in the article's Creative Commons licence, unless indicated otherwise in a credit line to the material. If material is not included in the article's Creative Commons licence and your intended use is not permitted by statutory regulation or exceeds the permitted use, you will need to obtain permission directly from the copyright holder. To view a copy of this licence, visit http://creativecommons.org/licenses/by/4.0/. The Creative Commons Public Domain Dedication waiver (http://creativeco mmons.org/publicdomain/zero/1.0/) applies to the data made available in this article, unless otherwise stated in a credit line to the data. 